\title{
Progression in the severity of aortic stenosis according to race among those with advanced chronic kidney disease
}

\author{
Aamir Husain ${ }^{1}$, Sameer Arora ${ }^{2,3}$, Paula D. Strassle ${ }^{3}$, Greg Means ${ }^{2}$, Chinmay Patel ${ }^{4}$, Thomas G. Caranasos ${ }^{2}$, \\ Alan L. Hinderliter ${ }^{2}$, John P. Vavalle ${ }^{2}$ \\ ${ }^{1}$ Division of Internal Medicine, University of Illinois at Chicago, Chicago, IL, USA; ${ }^{2}$ Division of Cardiology, ${ }^{3}$ Division of Epidemiology, Gillings \\ School of Public Health, University of North Carolina, Chapel Hill, NC, USA; ${ }^{4}$ Nephrology, Pikeville Medical Center, Pikeville, KY, USA \\ Contributions: (I) Conception and design: A Husain, S Arora, AL Hinderliter, JP Vavalle; (II) Administrative support: AL Hinderliter, JP Vavalle; \\ (III) Provision of study material or patients: AL Hinderliter; (IV) Collection and assembly of data: A Husain, PD Strassle; (V) Data analysis and \\ interpretation: PD Strassle; (VI) Manuscript writing: All authors; (VII) Final approval of manuscript: All authors. \\ Correspondence to: John P. Vavalle, MD, MHS. Division of Cardiology, University of North Carolina, 160 Dental Circle, Campus Box 7075, Chapel \\ Hill, NC 27599, USA. Email: john_vavalle@med.unc.edu.
}

Background: There is a higher prevalence of aortic stenosis (AS) in patients with advanced chronic kidney disease (CKD) and European ancestry. However, studies comparing AS progression in white and black patients in an advanced CKD population do not exist.

Methods: Advanced CKD (stage IV-V) patients who were referred to the UNC Cardiorenal Clinic for pre-operative kidney transplant evaluation, and diagnosed with either AS (mild, moderate, or severe) or a left ventricular outflow tract velocity $\geq 2 \mathrm{~m} / \mathrm{s}$ at any point between 2006-2016 were eligible for inclusion. Serial transthoracic echocardiograms over the 10-year period determined AS progression. All echocardiograms acquired after renal transplantation or aortic valve replacement were excluded. The rates of change of three indices of AS severity [mean gradient, aortic valve area (AVA), and aortic valve velocity] were compared between white and black patients. Mixed effects linear models with repeated measures were used to estimate the overall and race-stratified yearly rate of progression for each index, adjusted for age, sex, smoking status, dialysis, and baseline cholesterol.

Results: Of 1,283 patients, 140 (34\% white, 66\% black) developed or had baseline AS. Initially, $81 \%$ had no AS, $13 \%$ had mild, and $6 \%$ had moderate. White patients were more likely to be male and less likely to be on hemodialysis compared to black patients. No differences in AS severity $(\mathrm{P}=0.55)$ or age (60 vs. 58 years, $\mathrm{P}=0.34)$ were seen at baseline. In white $v s$. black patients, mean gradient increased at 1.90 (95\% CI: 0.79, 3.01) mmHg/year vs. 1.46 (95\% CI, 0.79, 2.14) mmHg/year, $\mathrm{P}=0.20$, AVA decreased at -0.10 (95\% CI: -0.15 , -0.05 ) $\mathrm{m}^{2} /$ year vs. -0.08 (95\% CI: $\left.-0.11,-0.05\right) \mathrm{m}^{2} /$ year, $\mathrm{P}=0.13$, and transvalvular velocity increased at 0.11 (95\% CI: 0.04, 0.18) m/s/year vs. 0.07 (95\% CI: 0.03, 0.11) m/s/year, P=0.09.

Conclusions: Compared to black patients, white patients in an advanced CKD cohort may have exhibited more rapid progression of AS. Ours is the first study to analyze racial differences in such a population. A study with a larger sample size is needed to confirm our findings.

Keywords: Aortic valve stenosis; chronic renal insufficiency; aortic valve

Submitted Apr 22, 2019. Accepted for publication Jun 13, 2019.

doi: $10.21037 /$ cdt.2019.06.04

View this article at: http://dx.doi.org/10.21037/cdt.2019.06.04 


\section{Introduction}

More than 17,000 deaths each year can be attributed to aortic valve disease (1). The mortality of patients with symptomatic severe aortic stenosis (AS) is as high as $50 \%$ at 1 year (2). European heritage and chronic kidney disease $(\mathrm{CKD})$ are associated with an increased risk of developing calcific AS (3-5). The higher prevalence of AS in those of European ancestry has been supported by limited genetic analysis with abundant data demonstrating a higher prevalence of AS in white adults, as well as increased ASassociated interventions in whites compared to black patients (5-7). Renal dysfunction has been hypothesized to promote aortic valve calcification leading to AS by inducing high rates of inflammation, oxidative stress, and derangements in calcium and phosphorus homeostasis (8). However, it is unknown if this association between CKD and AS blunts the strong relationship between race and calcification of the aortic valve. The aim of our study is to compare the progression of AS between whites and black patients in a CKD specific population and investigate if the genetic link between white adults and AS progression prevails even in a CKD population.

\section{Methods}

Patients with advanced CKD referred to the University of North Carolina Chapel-Hill Cardiorenal Clinic from 2006-2016 for pre-operative kidney transplant evaluation were examined for aortic valve disease by serial transthoracic echocardiography (TTE). Patients referred to this clinic were either on dialysis already or had a GFR $<25 \mathrm{~mL} / \mathrm{min} / 1.73 \mathrm{~m}^{2}$. Of this group, those with documented AS (mild, moderate, severe) either at baseline or over their 10-year trajectory by echocardiography report were included. In addition, those with a peak transvalvular velocity $\geq 2 \mathrm{~m} / \mathrm{s}$ at any point during this time were also included. Patients who did not have AS or a transvalvular velocity $\geq 2 \mathrm{~m} / \mathrm{s}$ on initial presentation were included if they developed either of these criteria on a subsequent echocardiogram. AS was defined through qualitative analysis of echocardiogram imaging with mild AS generally being defined as a peak transvalvular velocity of 2.0 to $2.9 \mathrm{~m} / \mathrm{s}$ or mean gradient $<20 \mathrm{mmHg}$, moderate AS being defined as a peak transvalvular velocity of 3.0 to $3.9 \mathrm{~m} / \mathrm{s}$ or mean gradient 20 to $39 \mathrm{mmHg}$, and severe AS being defined as a peak transvalvular velocity $\geq 4 \mathrm{~m} / \mathrm{s}$ or mean gradient of $\geq 40 \mathrm{mmHg}$ with an aortic valve area (AVA) typically $\leq 1 \mathrm{~cm}^{2}$. Given that a qualitative assessment was used to classify patients with AS, we included the additional parameter of peak transvalvular velocity $\geq 2 \mathrm{~m} / \mathrm{s}$ to capture additional patients with a predisposition to AS. Echocardiograms performed after aortic valve replacement or renal transplant were excluded from analysis, as were all transesophageal echocardiograms.

Ultrasound studies were performed in the UNC Hospitals Echocardiography Laboratory by trained sonographers using Philips Sonos 5500 and IE33 imaging platforms (Philips, Andover, MA, USA). A standardized, comprehensive imaging protocol that included detailed assessments of valvular and ventricular function was employed. Specifically, pulsed wave and continuous wave Doppler profiles of flow in the left ventricular outflow tract and through the aortic valve were acquired, allowing calculation of the peak transvalvular velocity, the estimated mean transvalvular gradient, and estimated AVA by the continuity equation. With few exceptions, all echocardiograms were read by the same faculty echocardiography reader, which provided consistency among interpretations.

All echocardiographic data was obtained through chart abstraction. Serial TTEs extrapolated over a 10 -year period were used to determine yearly rates of changes of three echocardiographic indices of AS severity, including mean transvalvular gradient, estimated AVA, and peak transvalvular velocity. Mean gradient and transvalvular velocity measures were excluded if the patient's ejection fraction was $<50 \%(n=20)$. All demographic and laboratory data were obtained at the patient's first visit to the cardiorenal clinic. Patients were followed from 2006 until March 2016. IRB approval was obtained through the University of North Carolina system and informed consent was appropriately obtained prior to study involvement.

Patient demographics and AS measurements (mean transvalvular gradient, estimated AVA, and peak transvalvular velocity) at the first visit were described using frequency $s$ and univariate analyses. If a patient's ejection fraction was $<50 \%$, their estimated mean gradient and aortic valve velocity values were excluded, but AVA was included in the analysis. Differences in demographics between patients were assessed using Fisher's exact and Wilcoxon-Mann-Whitney tests, where appropriate. A $\mathrm{P}$ value $<0.05$ was considered statistically significant.

A mixed effect linear model with repeated measures was used to estimate the average yearly rate of progression for the cohort. An unstructured correlation matrix was used to estimate the random effects of individual intercept and 
slope. For slope estimates, a positive value indicates an increase in measurement values over time and a negative value indicates a decrease. Multivariable mixed effect linear models, adjusting for age, sex, smoking status, dialysis, and baseline total cholesterols, were also performed. Age and total cholesterol were modeled as restricted quadratic splines.

Mixed effect linear regression with repeated measures was also used to determine if race (black $v s$. white) modified the rate of AS progression (i.e., change the slope of progression). Models were again adjusted for age, sex, smoking status, dialysis, and baseline total cholesterol, and interaction terms were used to assess whether significant differences in progression existed. All analyses were performed using SAS software version 9.4 (SAS Inc., Cary, NC, USA).

\section{Results}

Of the 1,283 patients from the cardiorenal clinic database, 148 individuals (93 black, 47 white, and 8 other race patients) met the inclusion criteria of having at least mild AS or a peak aortic transvalvular velocity of at least $2 \mathrm{~m} / \mathrm{sec}$ at any point during their follow-up trajectory, and at least one echocardiogram available for interpretation. This resulted in 408 eligible TTEs during the study period. The median number of visits was 2 (IQR, 1-4) and 101 patients (68\%) had at least 2 TTEs during the study period. Median time between eligible TTEs was 362 (IQR, 157-539) days among white patients and 434 (IQR, 250-616) days for black patients, $\mathrm{P}=0.03$. However, the median number of TTEs were the same between both groups (median 2, IQR, $1-4), P=0.83$. The study population was predominantly male (54\%), black (66\%), and had a median age of 58 (IQR, 51-64) years with $79 \%$ on either hemodialysis or peritoneal dialysis (Table 1). Diabetics comprised $56 \%$ of the population, $100 \%$ were hypertensive, and $20 \%$ had either mild or moderate AS at baseline. In addition, 28 patients had renal transplantation during the study period. At the initial visit, the average mean transvalvular gradient was 9 (IQR, 6-12) $\mathrm{mmHg}$, the average estimated AVA was 1.8 (IQR, 1.4-2.1) $\mathrm{cm}^{2}$, and the average transvalvular velocity was 2.0 (IQR, 1.8-2.3) m/s.

Compared to whites, black patients $(66 \%)$ were of similar age at first echocardiogram (58 vs. 60 years), more likely female (54\% vs. $30 \%, \mathrm{P}=0.008$ ), and more likely on dialysis (84\% vs. 66\%, $\mathrm{P}=0.01$ ) (Table 1). Diabetes (59\% vs. 49\%, $\mathrm{P}=0.28)$, smoking ( $43 \%$ vs. $60 \%, \mathrm{P}=0.07)$, hypertension (100\% vs. $100 \%, \mathrm{P}=0.99$ ), and phosphorus levels (5.2, IQR, 4.5-6.2; vs. 5.0, IQR, 4.1-5.7, $\mathrm{P}=0.15$ ) were similar between the two groups. Total cholesterol (175, IQR, 145-212; vs. 148, IQR, 124-175, $\mathrm{P}=0.02)$ and HDL cholesterol (47, IQR, 39-59; vs. 40, IQR, 32-52) were higher in black compared to white patients. At baseline, there was no difference in the prevalence of AS between black and white patients (24\% vs. $15 \%, \mathrm{P}=0.55)$. There was also no significant difference in baseline values of the 3 AS indices between the two groups.

With regards to overall progression, mean transvalvular gradient increased at $1.26 \mathrm{mmHg}$ /year (95\% CI: 0.64 , 1.88), estimated AVA decreased at -0.07 (95\% CI: -0.09 , $-0.04) \mathrm{m}^{2} /$ year, and peak transvalvular velocity increased at $0.06(95 \%$ CI: $0.02,0.10) \mathrm{m} / \mathrm{s} /$ year (Table 2). Minimal differences were seen after accounting for age, sex, smoking status, dialysis, and baseline total cholesterol (Table 2). In white $v s$. black patients, mean gradient increased at 1.90 (95\% CI: 0.79, 3.01) mmHg/year vs. 1.46 (95\% CI, 0.79, 2.14) $\mathrm{mmHg} /$ year, $\mathrm{P}=0.20$, AVA decreased at $-0.10(95 \%$ CI: $-0.15,-0.05) \mathrm{m}^{2} /$ year $v s .-0.08$ (95\% CI: $\left.-0.11,-0.05\right)$ $\mathrm{m}^{2} /$ year, $\mathrm{P}=0.13$, and transvalvular velocity increased at 0.11 (95\% CI: 0.04, 0.18) m/s/year vs. 0.07 (95\% CI: 0.03, 0.11 ) $\mathrm{m} / \mathrm{s} /$ year, $\mathrm{P}=0.09$ (Table 3). Rates of progression in white $v$ s. black patients are plotted as slopes in Figure 1. An example of AS progression via echocardiogram imaging in an example patient is shown in Figure 2.

\section{Discussion}

In this population of 148 advanced CKD patients being evaluated for kidney transplantation, black patients were more likely to be female and on dialysis. Despite this, AS appears to have progressed more rapidly in white patients compared to black patients across all three AS indices. The rate of progression was at least two-fold higher in the white population for each index, despite a higher prevalence of dialysis in the black patients in our cohort. To our knowledge, our study is the first to compare AS progression by race in an advanced CKD population.

In this study on an advanced CKD population being evaluated for renal transplantation, we found black adults to more likely be on dialysis as compared to whites. The racerelationship is well supported in literature, as black patients are almost twice as likely to develop end-stage renal disease than whites after adjusting for common comorbidities and socioeconomic status $(9,10)$. This relationship holds true despite a similar prevalence of kidney disease at earlier CKD stages, suggesting a more rapid rate of kidney function decline in black patients compared to whites $(11,12)$.

Despite a lower proportion of white patients being 
Table 1 Characteristics of patients with advanced CKD and aortic stenosis at their first ECHO visit (baseline), stratified by race

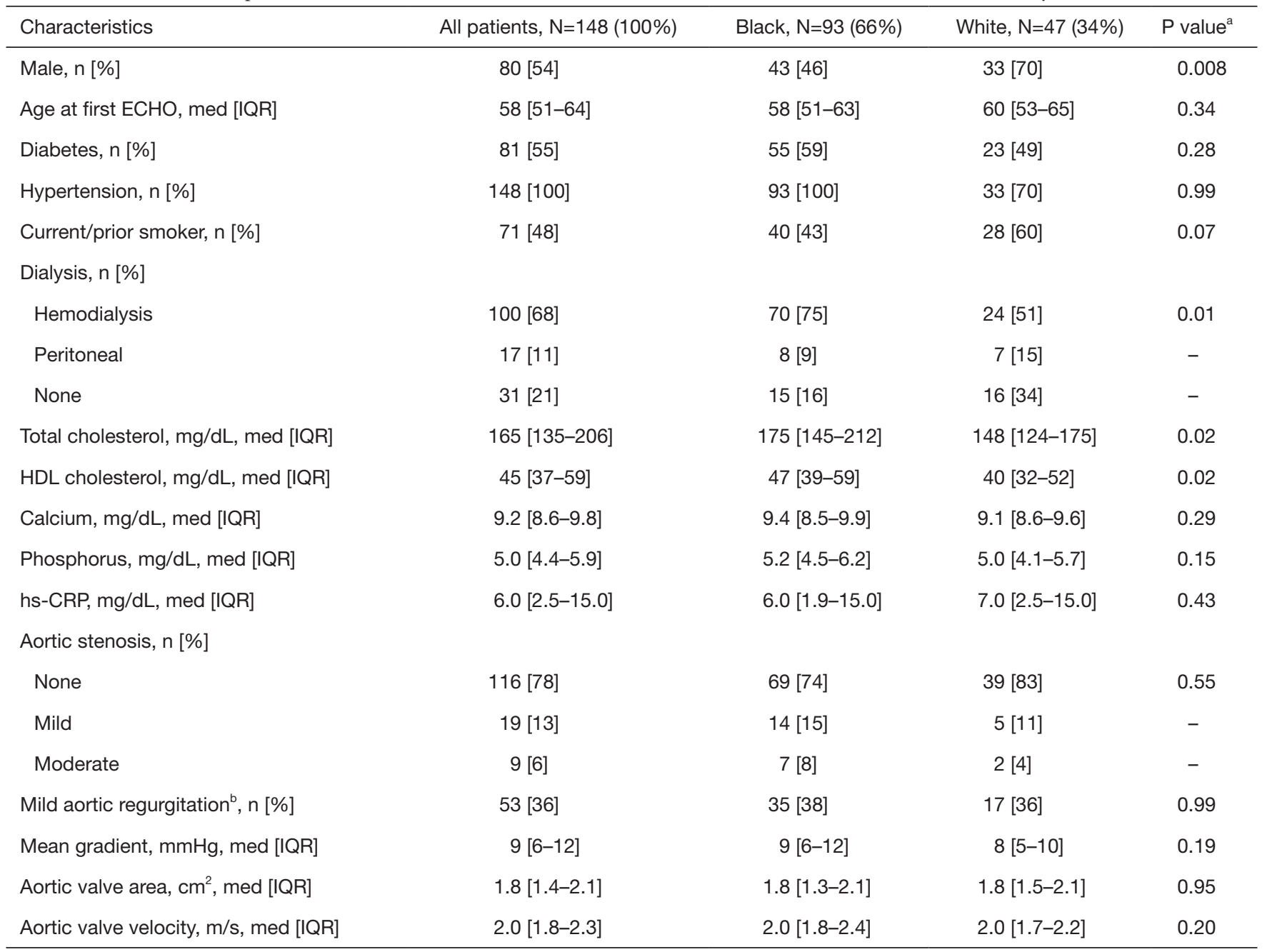

${ }^{a}$, Fisher's exact or Wilcoxon test comparing Black and White patients; ${ }^{b}$, compared to no aortic regurgitation. CKD, chronic kidney disease; med, median; IQR, interquartile range; HDL, high density lipoprotein; hs-CRP, high sensitivity C-reactive protein.

Table 2 Average, yearly rate of progression of aortic stenosis measures among patients with advanced CKD

\begin{tabular}{lcc}
\hline \multirow{2}{*}{ Variable } & \multicolumn{2}{c}{ CIE $(95 \% \mathrm{Cl})$} \\
\cline { 2 - 3 } & Crude $^{\mathrm{a}}$ & Adjusted $^{\mathrm{a}, \mathrm{b}}$ \\
\hline Mean gradient, $\mathrm{mmHg}$ & $1.27(0.67,1.87)$ & $1.26(0.64,1.88)$ \\
Aortic valve area, $\mathrm{cm}^{2}$ & $-0.07(-0.10,-0.04)$ & $-0.07(-0.09,-0.04)$ \\
Aortic valve velocity, & $0.06(0.02,0.10)$ & $0.06(0.02,0.10)$ \\
$\mathrm{m} / \mathrm{s}$ & & \\
\hline
\end{tabular}

a , time, in years, was modeled as a linear variable; an unstructured covariance for the random effects of intercept and slope was used; ${ }^{\text {b }}$, adjusted for sex, age, smoking status, dialysis, and total cholesterol at baseline; age and total cholesterol were modeled as restricted quadratic splines. CKD, chronic kidney disease. on dialysis, we found a significantly faster rate of AS progression in that group as compared to black patients. This potentially indicates a stronger role for genetic predisposition in AS progression as compared to advanced CKD. The fact that CKD accelerates AS progression has been previously demonstrated. Kim et al. showed that mild to moderate AS progressed faster in a population of end-stage renal disease patients compared to age and gender matched controls due to a higher hemodynamic and metabolic burden on the aortic valve (13). The higher metabolic burden leading to faster AS progression was also shown in a study by Kume et al., who found that greater calcification of the aortic valve in dialysis patients led to 
Table 3 Average yearly rate of progression of aortic stenosis measures among patients with advanced CKD, stratified by race

\begin{tabular}{|c|c|c|c|c|c|c|}
\hline Variable & \multicolumn{3}{|c|}{ Crude $^{a}$} & \multicolumn{3}{|c|}{ Adjusted $^{\mathrm{a}, \mathrm{b}}$} \\
\hline Mean gradient, $\mathrm{mmHg}$ & $1.45(0.81,2.09)$ & $1.87(0.81,2.94)$ & 0.20 & $1.46(0.79,2.14)$ & $1.90(0.79,3.01)$ & 0.20 \\
\hline Aortic valve area, $\mathrm{cm}^{2}$ & $-0.06(-0.08,-0.05)$ & $-0.10(-0.15,-0.05)$ & 0.12 & $-0.08(-0.11,-0.05)$ & $-0.10(-0.15,-0.05)$ & 0.13 \\
\hline Aortic valve velocity, $\mathrm{m} / \mathrm{s}$ & $0.07(0.03,0.07)$ & $0.11(0.04,0.18)$ & 0.07 & $0.07(0.03,0.11)$ & $0.11(0.04,0.18)$ & 0.09 \\
\hline
\end{tabular}

${ }^{a}$, time, in years, was modeled as a linear variable; an unstructured covariance for the random effects of intercept and slope was used;

b, adjusted for sex, age, smoking status, dialysis, and total cholesterol at baseline; age and total cholesterol were modeled as restricted quadratic splines; ${ }^{c}$, interaction test assessing whether difference between average yearly rate of aortic stenosis progression is statistically significant between black and white patients. CKD, chronic kidney disease.
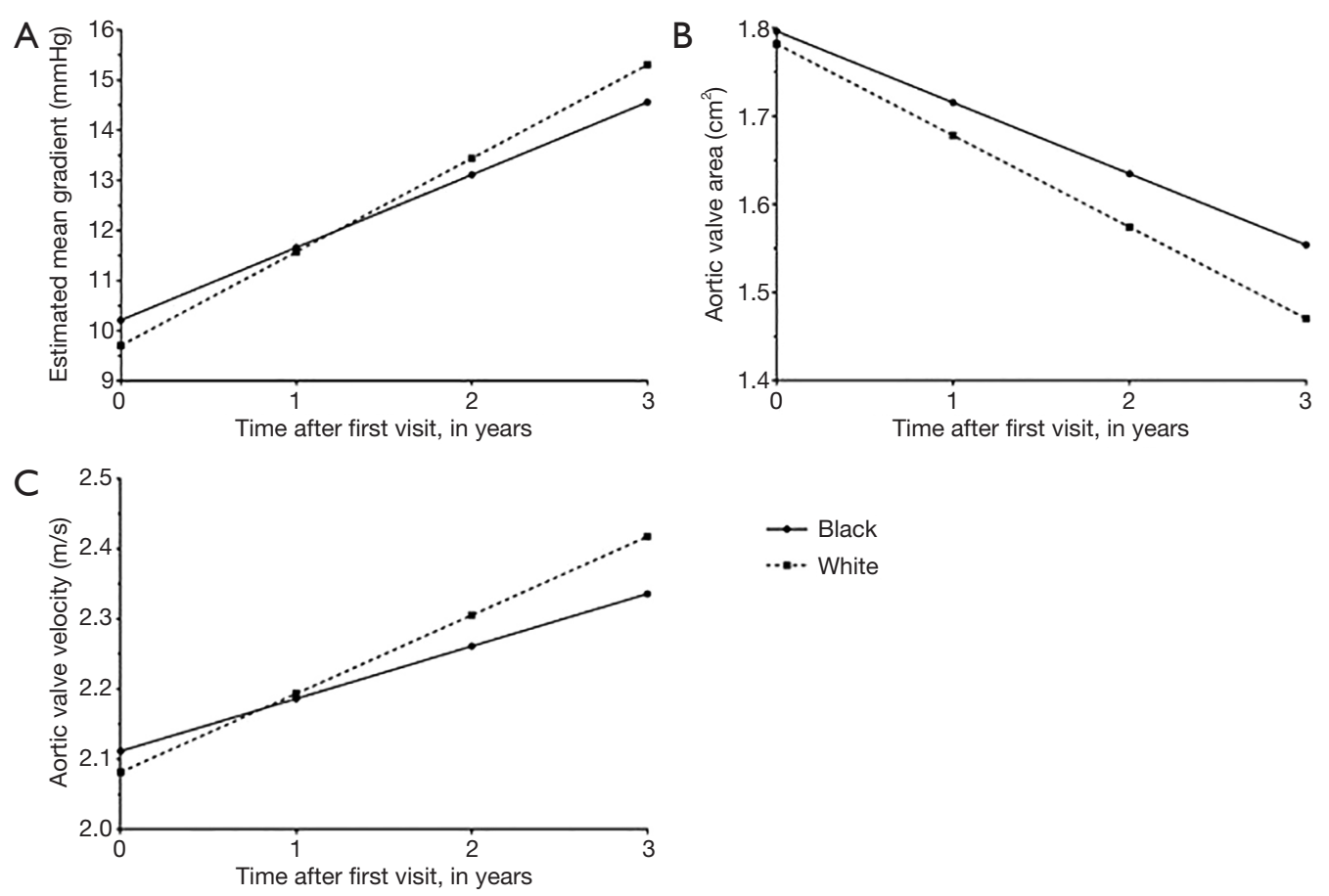

Figure 1 Rates of change of 3 AS indices in White $v s$. Black patients over time. (A) Estimated change in mean gradient (mmHg) over time; (B) estimated change in aortic valve area $\left(\mathrm{cm}^{2}\right)$ over time; (C) estimated change in aortic valve velocity ( $\left.\mathrm{m} / \mathrm{s}\right)$ over time; solid line represents black patients with the dotted line representing white patients. AS, aortic stenosis.

worsened AS more quickly (14). CKD is a risk factor for worsened AS severity even in pre stage 5 CKD populations, suggesting that even a moderate loss of kidney function results in worsening AS (15). This suggests that even in light of the metabolic and hemodynamic derangements present with advanced CKD, genetic predispositions still predominate AS progression.

The relationship between European ancestry and AS progression may be due to single nucleotide polymorphisms associated with aortic valve calcification. Thanassoulis and colleagues were able to demonstrate a strong association between the lipoprotein (a) (LPA) locus and the presence of aortic valve calcification in patients of European descent (5). Although this association was only based on valve calcification, it may be reasonable to extrapolate this association between LPA AS and AS progression as well. Ellis et al. located another 3 single nucleotide polymorphisms associated with AS in patients older than 60 who were $>98 \%$ Caucasian (16). A further understanding of the genetics underlying race differences in valvular disease 

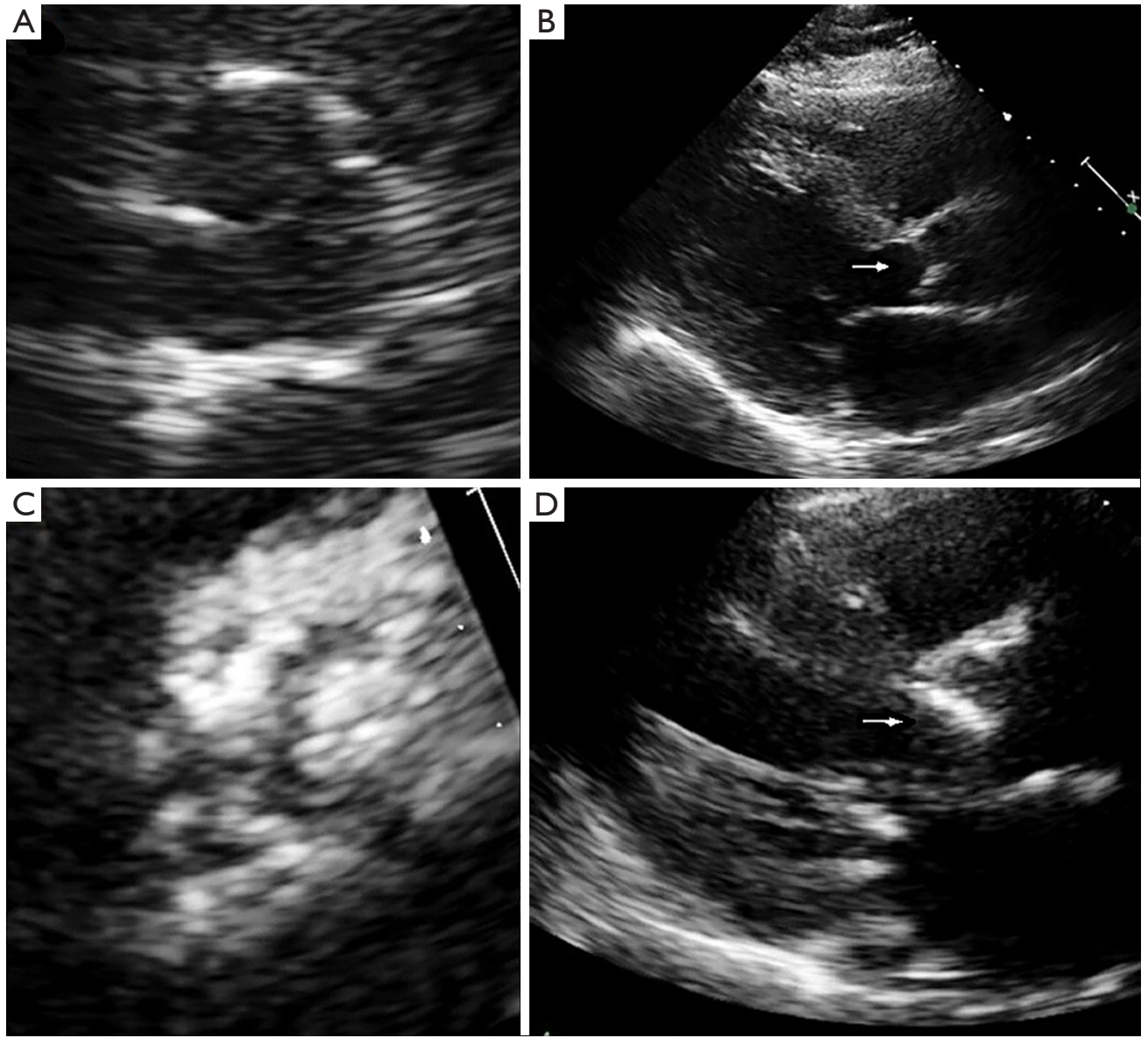

Figure 2 Images of AS progression over time via echocardiography in an example patient. (A) Short axis view of the aortic valve at baseline; (B) long axis view of the aortic valve (arrow) at baseline; (C) short axis view of the aortic valve 5 years later; (D) long axis view of the aortic valve (arrow) 5 years later. The bright white echo dense areas on the valve represent calcification. AS, aortic stenosis.

is necessary to make definitive conclusions.

Another possible explanation may relate to the relationship observed in overall decreased levels of coronary calcification in black patients compared to whites. The Multi-Ethnic Study of Atherosclerosis (MESA) showed that in a cohort aged 45 to 84 years without clinical coronary artery disease, white patients had rates of coronary calcification up to $22 \%$ higher than corresponding black patients after adjustment for coronary risk factors (17). It is possible that the predisposing factors that promoted more aggressive coronary calcification in white patients were similar to those promoting aortic valve calcification and thus AS. Differences in vitamin D metabolism between white and black patients may possibly explain the variations in calcification between them (18).

This study is not without limitations. First, this is a single center study with a modest sample size. However, our results are indicative of the fact that meaningful differences in AS progression between white and black patients may exist. Second, the relatively young age of our cohort almost certainly contributed to slower rates of AS progression than that of the general population. This is particularly important as the severity of valvular stenosis is known to increase considerably with age, especially after 75 years (19). Additionally, our population at baseline largely had mild AS as opposed to moderate or severe, possibly reducing the rate of progression. Lastly, we understand that the pre-transplant evaluation is rigorous and likely excludes otherwise sicker patients in the general CKD population. This is in conjunction with the fact that mineral bone disorder is likely managed differently in pretransplant patients. 


\section{Conclusions}

In this study investigating 148 advanced CKD patients who underwent serial echocardiography over a 10 -year period, AS appears to progress at a faster pace in white patients compared to black patients in each of the three AS indices (mean gradient, AVA, transvalvular velocity). These results suggest that the genetic predisposition for AS may be a strong risk factor for AS progression. Additional research is required to confirm the results of our study.

\section{Acknowledgments}

None.

\section{Footnote}

Conflicts of Interest: The authors have no conflicts of interest to declare.

Ethical Statement: IRB approval was obtained through the University of North Carolina system and informed consent was appropriately obtained prior to study involvement. The authors are accountable for all aspects of the work in ensuring that questions related to the accuracy or integrity of any part of the work are appropriately investigated and resolved.

\section{References}

1. Benjamin EJ, Blaha MJ, Chiuve SE, et al. Heart Disease and Stroke Statistics'2017 Update: A Report from the American Heart Association. Circulation 2017;135:e146-603.

2. Leon MB. Transcatheter aortic-valve implantation for aortic stenosis in patients who cannot undergo surgery. $\mathrm{N}$ Engl J Med 2010;363:1597-607.

3. Straumann E, Meyer B, Misteli M, et al. Aortic and mitral valve disease in patients with end stage renal failure on long-term haemodialysis. Br Heart J 1992;67:236-9.

4. Zentner D, Hunt D, Chan W, et al. Prospective evaluation of aortic stenosis in end-stage kidney disease: A more fulminant process? Nephrol Dial Transplant 2011;26:1651-5.

5. Thanassoulis G, Campbell CY, Owens DS, et al. Genetic associations with valvular calcification and aortic stenosis. N Engl J Med 2013;368:503-12.

6. Yeung M, Kerrigan J, Sodhi S, et al. Racial differences in rates of aortic valve replacement in patients with severe aortic stenosis. Am J Cardiol 2013;112:991-5.

7. Patel DK, Green KD, Fudim M, et al. Racial differences in the prevalence of severe aortic stenosis. J Am Heart Assoc 2014;3:e00879.

8. Tarrass F, Benjelloun M, Zamd M, et al. Heart valve calcifications in patients with end-stage renal disease: Analysis for risk factors. Nephrology 2006;11:494-6.

9. Parsa A, Kao WHL, Xie D, et al. APOL1 Risk variants, race, and progression of chronic kidney disease. $\mathrm{N}$ Engl J Med 2013;369:2183-96.

10. Lipworth L, Mumma MT, Cavanaugh KL, et al. Incidence and predictors of end stage renal disease among lowincome blacks and whites. PLoS One 2012;7:e48407.

11. Hsu CY. Racial differences in the progression from chronic renal insufficiency to end-stage renal disease in the United States. J Am Soc Nephrol 2003;14:2902-7.

12. Choi AI, Rodriguez RA, Bacchetti P, et al. White/black racial differences in risk of end-stage renal disease and death. Am J Med 2009;122:672-8.

13. Kim D, Shim CY, Hong GR, et al. Effect of end-stage renal disease on rate of progression of aortic stenosis. Am J Cardiol 2016;117:1972-7.

14. Kume T, Kawamoto T, Akasaka T, et al. Rate of progression of valvular aortic stenosis in patients undergoing dialysis. J Am Soc Echocardiogr 2006;19:914-8.

15. Masuda C, Dohi K, Sakurai Y, et al. Impact of chronic kidney disease on the presence and severity of aortic stenosis in patients at high risk for coronary artery disease. Cardiovasc Ultrasound 2011;9:31.

16. Ellis SG, Dushman-Ellis S, Luke MM, et al. Pilot candidate gene analysis of patients $<60$ years old with aortic stenosis involving a tricuspid aortic valve. Am J Cardiol 2012;110:88-92.

17. Bild DE, Detrano R, Peterson D, et al. Ethnic differences in coronary calcification: The Multi-Ethnic Study of Atherosclerosis (MESA). Circulation 2005;111:1313-20.

18. Doherty TM, Tang W, Dascalos S, et al. Ethnic origin and serum levels of 1 $\alpha, 25$-Dihydroxyvitamin D3 are independent predictors of coronary calcium mass measured by electronbeam computed tomography. Circulation. 1997;96:1477-81.

19. Ohara T, Hashimoto Y, Matsumura A, et al. Accelerated progression and morbidity in patients with aortic stenosis on chronic dialysis. Circ J 2005;69:1535-9.

Cite this article as: Husain A, Arora S, Strassle PD, Means G, Patel C, Caranasos TG, Hinderliter AL, Vavalle JP. Progression in the severity of aortic stenosis according to race among those with advanced chronic kidney disease. Cardiovasc Diagn Ther 2020;10(1):24-30. doi: 10.21037/cdt.2019.06.04 